\title{
Divergent $\Rightarrow$ complex amplitudes in two dimensional string theory
}

\author{
Ashoke Sen \\ Harish-Chandra Research Institute, HBNI, \\ Chhatnag Road, Jhusi, Allahabad 211019, Indi \\ E-mail: sen@hri.res.in
}

ABSTRACT: In a recent paper, Balthazar, Rodriguez and Yin found remarkable agreement between the one instanton contribution to the scattering amplitudes of two dimensional string theory and those in the matrix model to the first subleading order. The comparison was carried out numerically by analytically continuing the external energies to imaginary values, since for real energies the string theory result diverges. We use insights from string field theory to give finite expressions for the string theory amplitudes for real energies. We also show analytically that the imaginary parts of the string theory amplitudes computed this way reproduce the full matrix model results for general scattering amplitudes involving multiple closed strings.

Keywords: M(atrix) Theories, String Field Theory

ARXIV EPRINT: 2003.12076 


\section{Contents}

1 Introduction and summary 1

2 Scattering involving multiple closed string tachyons 4

$\begin{array}{lll}3 & \text { Divergences to imaginary parts } & 6\end{array}$

\section{Introduction and summary}

D-instantons represent saddle points of the path integral in second quantized string theory and give non-perturbative contribution to the string amplitudes. The usual world-sheet approach to computing these corrections suffer from infra-red divergences from the boundary of the moduli spaces of Riemann surfaces. A concrete example of such ambiguities arose in a recent analysis of two dimensional string theory [1]. The authors of [1] computed the D-instanton contribution to the closed string scattering amplitude, but could determine the final result only up to two undetermined constants. Furthermore, the string theory result diverges for real energies of the external states, and so in order to get finite results, [1] evaluated the string theory results for imaginary energies of the external states. These results were then compared numerically with the results in the dual matrix model [2-5], leading to the best fit values -1.399 and .496 for the undertermined constants. ${ }^{1}$

String field theory [7-9] is well poised to address the issues related to infra-red divergences arising in string theory [9-11]. It does so by drawing insights from quantum field theory. Indeed, in an earlier analysis [12], string field theory was used to determine the first of these constants unambiguously, leading to the value $-\ln 4$, which is within $1 \%$ of the numerical result -1.399 . The second constant can also be fixed by this procedure [13]. Both of these ambiguities arise from divergences in the open string channel, where one or more of the internal open string states go on-shell or become tachyonic. In this paper we address the problem of evaluation of the amplitude for real energies of external states. As will be explained below, the associated divergences arise from internal closed string states going on-shell. However string field theory can be used to address these divergences as well.

We shall now summarize the main results, leaving the actual derivation of the results to section 2 and section 3 . We begin with a review of the results of [1] in which the authors computed the D-instanton contribution to the $1 \rightarrow 1$ scattering amplitude of closed string tachyon in two dimensional string theory to first subleading order in the expansion in the

\footnotetext{
${ }^{1}$ Ref. [6] extended this analysis to multi-instanton contribution. However since the analysis was done only for the leading order terms in the expansion in powers of the string coupling around the multi-instanton solution, the ambiguities of this type did not arise in [6].
} 
string coupling constant $g_{s}$. Their result takes the form:

$$
\begin{aligned}
A_{1 \rightarrow 1}= & 4 \mathcal{N} e^{-1 / g_{s}} 2 \pi \delta\left(\omega_{1}+\omega_{2}\right) \sinh \left(\pi\left|\omega_{1}\right|\right) \sinh \left(\pi\left|\omega_{2}\right|\right) \\
& {\left[1+g_{s} f\left(\omega_{1}, \omega_{2}\right)+g_{s} g\left(\omega_{1}\right)+g_{s} g\left(\omega_{2}\right)+C g_{s}+\mathcal{O}\left(g_{s}^{2}\right)\right], }
\end{aligned}
$$

where $-\omega_{1}>0$ represents the energy of the incoming tachyon, $\omega_{2}>0$ denotes the energy of the outgoing tachyon, $\mathcal{N}$ is a normalization constant, $C$ is a constant, and

$$
\begin{aligned}
f\left(\omega_{1}, \omega_{2}\right)= & 2^{-1 / 4} \pi^{1 / 2} 2^{\left(\omega_{1}^{2}+\omega_{2}^{2}\right) / 2} \frac{1}{\sinh \left(\pi\left|\omega_{1}\right|\right) \sinh \left(\pi\left|\omega_{2}\right|\right)} \int_{0}^{1} d y \\
& y^{\omega_{2}^{2} / 2}(1-y)^{1-\omega_{1} \omega_{2}}(1+y)^{1+\omega_{1} \omega_{2}}\left\langle V_{\left|\omega_{1}\right| / 2}(i) V_{\left|\omega_{2}\right| / 2}(i y)\right\rangle_{D}, \\
g(\omega)= & 2 \pi^{2} \frac{1}{\sinh (\pi|\omega|)} \int_{0}^{\infty} d t \int_{0}^{1 / 4} d x \eta(i t)\left(\frac{2 \pi}{\theta_{1}^{\prime}(0 \mid i t)} \theta_{1}(2 x \mid i t)\right)^{\omega^{2} / 2}\left\langle V_{|\omega| / 2}(2 \pi x)\right\rangle_{A} .
\end{aligned}
$$

$\theta_{1}(z \mid \tau)$ is the odd Jacobi theta function and $\theta_{1}^{\prime}(z \mid \tau) \equiv \partial_{z} \theta_{1}(z \mid \tau) .\left\langle V_{\left|\omega_{1}\right| / 2}(i) V_{\left|\omega_{2}\right| / 2}(i y)\right\rangle_{D}$ denotes the two point function on the upper half plane of a pair of primaries in the $\mathrm{c}=25$ Liouville theory, carrying momenta $\left|\omega_{1}\right| / 2$ and $\left|\omega_{2}\right| / 2$, inserted at $i$ and $i y$ respectively. $\left\langle V_{|\omega| / 2}(2 \pi x)\right\rangle_{A}$ denotes the one point function of the Liouville primary of momentum $|\omega| / 2$ on an annulus described by $0 \leq \operatorname{Re}(w) \leq \pi, w \equiv w+2 \pi i t$, with the vertex operator inserted at $\operatorname{Re}(w)=2 \pi x$. Explicit expressions for these correlation functions can be found in [1]. In (1.1), inside the square bracket, the leading term 1 represents the product of two disk one point functions of the closed string tachyons. The subleading terms proportional to $g_{s}$ come from three sources. The term proportional to $f\left(\omega_{1}, \omega_{2}\right)$ represents the contribution from the disk two point function of closed string tachyon vertex operators. The terms proportional to $g\left(\omega_{1}\right)$ and $g\left(\omega_{2}\right)$ represent the product of one point function on the disk and one point function on the annulus of the closed string tachyon. Finally the term involving the constant $C$ is the contribution from the product of the two disk one point functions and the zero point function on surfaces of Euler number $-1-$ a torus with a hole and a disk with two holes. The normalization constant $\mathcal{N}$ was fixed by comparison with the matrix model result to be [1]:

$$
\mathcal{N}=-\frac{1}{8 \pi^{2}}
$$

In the analysis of this paper we shall use (1.4) without proving it.

The integral (1.2) defining $f\left(\omega_{1}, \omega_{2}\right)$ diverges from the $y \simeq 0$ and $y \simeq 1$ regions, while the integral (1.3) defining $g(\omega)$ diverges from the $t \simeq \infty, x \simeq 0$ and $t \simeq 0$ regions. The divergence of $f$ from the $y \simeq 0$ region and that of $g$ from the $t \simeq \infty$ and $x \simeq 0$ regions are associated with open string degenerations, and string field theory can be used to get unambiguous finite results for the integrals from this region [12-14], fixing the two undetermined constants in the analysis of [1]. Ref. [1] dealt with the divergences associated with the $y \simeq 1$ and $t \simeq 0$ regions, associated with the closed string degeneration, by working with imaginary energies. In section 3 we shall use insights from string field theory to deal with these divergences, and describe the procedure for getting finite results for these 
integrals for real energies. The main idea follows the one described in [15], - to represent the amplitudes as Feynman diagrams of string field theory, and carry out the integrals over momentum variables along appropriate contours in the complex momentum plane. In fact we do not need to go through the analysis is detail, but simply lift the results of [15] to the case under study. ${ }^{2}$

Once we have divergence free expressions for $f\left(\omega_{1}, \omega_{2}\right)$ and $g(\omega)$, we can also use them to compute a general $n+1$-point amplitude in which an incoming closed string tachyon of energy $-\omega_{n+1}>0$ scatters to $n$ outgoing closed string tachyons of energies $\omega_{1}, \cdots, \omega_{n}>0$. The result takes the form:

$$
\begin{aligned}
A_{1 \rightarrow n}= & 2^{n+1} \mathcal{N} e^{-1 / g_{s}} 2 \pi \delta\left(\omega_{1}+\omega_{2}+\cdots \omega_{n}+\omega_{n+1}\right)\left\{\prod_{i=1}^{n+1} \sinh \left(\pi\left|\omega_{i}\right|\right)\right\} \\
& {\left[1+g_{s} \sum_{\substack{i, j=1 \\
i<j}}^{n+1} f\left(\omega_{i}, \omega_{j}\right)+g_{s} \sum_{i=1}^{n+1} g\left(\omega_{i}\right)+C g_{s}\right] . }
\end{aligned}
$$

The matrix model result for this amplitude can be computed using the ingredients given in [1]. This has been described in section 2 and the result takes the form:

$$
\begin{aligned}
2^{n+1} \mathcal{N} e^{-1 / g_{s}} 2 \pi \delta\left(\omega_{1}+\omega_{2}+\cdots \omega_{n}+\omega_{n+1}\right)\left\{\prod_{i=1}^{n+1} \sinh \left(\pi\left|\omega_{i}\right|\right)\right\} \\
{\left[1-i g_{s} \sum_{j=1}^{n} \omega_{j}\left(1-\sum_{i=1}^{n} \pi \omega_{i} \operatorname{coth}\left(\pi \omega_{i}\right)\right)\right] . }
\end{aligned}
$$

Equality of (1.5) and (1.6) demands the following identity:

$$
\begin{aligned}
& \sum_{\substack{i, j=1 \\
i<j}}^{n} f\left(\omega_{i}, \omega_{j}\right)+\sum_{i=1}^{n} f\left(\omega_{i},-\omega_{1}-\cdots-\omega_{n}\right)+\sum_{i=1}^{n} g\left(\omega_{i}\right)+g\left(-\omega_{1}-\cdots-\omega_{n}\right)+C \\
= & -i \sum_{j=1}^{n} \omega_{j}\left(1-\sum_{i=1}^{n} \pi \omega_{i} \operatorname{coth}\left(\pi \omega_{i}\right)\right), \quad \text { for } \omega_{i}>0,1 \leq i \leq n .
\end{aligned}
$$

Note that $f$ and $g$ given in (1.2) and (1.3) are formally real, in the sense that the integrands appearing in their expressions are real. On the other hand, the matrix model answer, encoded in the right hand side of (1.7), is purely imaginary. This is a standard problem in the world-sheet approach to string theory [17-23], and whenever an amplitude is expected to acquire an imaginary part, the corresponding integral over the moduli space of Riemann surfaces diverge. The relevant divergences in this case arise from the closed string channel, - precisely those associated with the $y \simeq 1$ region for $f\left(\omega_{1}, \omega_{2}\right)$ and the $t \simeq 0$ regions for $g(\omega) .{ }^{3}$ Our analysis in section 3 , that shows how to extract finite results

\footnotetext{
${ }^{2}$ An alternative approach is to deform the contour of integration over the moduli of Riemann surfaces into complex plane [16].

${ }^{3}$ The divergences associated with the open string degeneration produces real result after being treated using string field theory techniques of [12-14].
} 
for both $f\left(\omega_{1}, \omega_{2}\right)$ and $g(\omega)$, does contain imaginary parts. In fact the imaginary parts turn out to have compact analytic expressions, given by: ${ }^{4}$

$$
\begin{aligned}
f_{\text {imaginary }}\left(\omega_{1}, \omega_{2}\right) & =\frac{1}{2} i \pi \omega_{1} \omega_{2}\left\{\operatorname{coth}\left(\pi \omega_{1}\right)+\operatorname{coth}\left(\pi \omega_{2}\right)\right\} \operatorname{sign}\left(\omega_{1}+\omega_{2}\right), \\
g_{\text {imaginary }}(\omega) & =\frac{i \pi}{2}|\omega|\left\{\omega \operatorname{coth}(\pi \omega)-\frac{1}{\pi}\right\} .
\end{aligned}
$$

It is easy to check that the imaginary part of the left hand side of (1.7), computed with (1.8) and (1.9), agrees with the right hand side of (1.7). This gives an analytical proof of the imaginary part of (1.7).

\section{Scattering involving multiple closed string tachyons}

Ref. [1] computed the following D-instanton induced amplitudes in two dimensional string theory. The disk one point function of a closed string tachyon of energy $\omega$ is given by

$$
2 \sinh (\pi|\omega|)
$$

In this and in all the following expressions the energies will always be taken to be outgoing, with an incoming particle regarded as carrying negative energy. In this notation, the disk two point function of a pair of closed string tachyons of energies $\omega_{1}$ and $\omega_{2}$ is given by

$$
4 g_{s} \sinh \left(\pi\left|\omega_{1}\right|\right) \sinh \left(\pi\left|\omega_{2}\right|\right) f\left(\omega_{1}, \omega_{2}\right),
$$

where $f\left(\omega_{1}, \omega_{2}\right)$ has been defined in (1.2). An annulus one point function of a closed string tachyon of energy $\omega$ is given by:

$$
2 g_{s} \sinh (\pi|\omega|) g(\omega)
$$

where $g(\omega)$ has been defined in (1.3). We shall also denote by $C g_{s}$ the zero point function on surfaces of Euler number -1. Each amplitude carries an overall normalization of $\mathcal{N} e^{-1 / g_{s}}$ where $\mathcal{N}$ is the normalization constant given in (1.4). Finally, the integration over the collective modes of the D-instanton generates a factor of $2 \pi \delta(\omega)$ where $\omega$ is the total energy carried by all the external states of the amplitude. Multiplication by $\mathcal{N} e^{-1 / g_{s}}$ and the $2 \pi \delta(\omega)$ factors has to be done at the end after taking the products of all the disconnected parts that an amplitude may have.

Using these results we can compute a general D-instanton induced scattering amplitude in which an incoming closed string tachyon of energy $-\omega_{n+1}>0$ scatters to $n$ outgoing closed string tachyons of energies $\omega_{1}, \cdots \omega_{n}$. The leading order term comes from the product of $n+1$ disk one point functions, each giving a factor of $2 \sinh \left(\pi\left|\omega_{i}\right|\right)$, while the subleading term is given by the sum of three kinds of diagrams - product of $(n-1)$ disk one point

\footnotetext{
${ }^{4}$ I wish to thank Bruno Balthazar, Victor Rodriguez and Xi Yin for raising the possibility of getting the imaginary parts of these amplitudes using unitarity cuts. Even though we did not use this, with hindsight one can see that the imaginary parts could have been obtained using the Cutkosky rules of string field theory [24]. The approach discussed in section 3 gives finite expressions for both, the real and imaginary parts of the amplitude, for real energies.
} 
functions and a disk two point function, the product of $n$ disk one point functions and an annulus one point function and the product of $n+1$ disk one point functions and a zero point function on a Riemann surface of Euler number -1. Therefore the result takes the form:

$$
\begin{aligned}
A_{1 \rightarrow n}= & 2^{n+1} \mathcal{N} e^{-1 / g_{s}} 2 \pi \delta\left(\omega_{1}+\omega_{2}+\cdots \omega_{n}+\omega_{n+1}\right)\left\{\prod_{i=1}^{n+1} \sinh \left(\pi\left|\omega_{i}\right|\right)\right\} \\
& {\left[1+g_{s} \sum_{\substack{i, j=1 \\
i<j}}^{n+1} f\left(\omega_{i}, \omega_{j}\right)+g_{s} \sum_{i=1}^{n+1} g\left(\omega_{i}\right)+C g_{s}\right] . }
\end{aligned}
$$

The matrix model result for $A_{1 \rightarrow n}$ was computed in [1]. There it was shown that the leading order result agrees with the leading order term in (2.4) (given by the 1 inside the square bracket) if we choose $\mathcal{N}$ as in (1.4). The subleading order result of the matrix model takes the form [1]:

$$
A^{(1)}=-\delta\left(\omega_{1}+\cdots \omega_{n+1}\right) e^{-1 / g_{s}} 2 \pi i g_{s} \omega \sinh (\pi \omega) \sum_{S}(-1)^{|S|} \int_{0}^{\omega_{S}} d x e^{\pi(\omega-2 x)}\left(x-\frac{\omega}{2}\right),
$$

where

$$
\omega \equiv \omega_{1}+\cdots+\omega_{n},
$$

$S$ is a subset of $\{1, \cdots, n\},|S|$ is the number of elements of $S$, and

$$
\omega_{S}=\sum_{i \in S} \omega_{i}
$$

After performing the integration over $x$ and summing over $S$ using the results,

$$
\begin{aligned}
\sum_{S}(-1)^{|S|} e^{-2 \pi \omega_{S}} & =\prod_{i=1}^{n}\left(1-e^{-2 \pi \omega_{i}}\right) \\
\sum_{S}(-1)^{|S|} \omega_{S} e^{-2 \pi \omega_{S}} & =-\prod_{i=1}^{n}\left(1-e^{-2 \pi \omega_{i}}\right) \sum_{j=1}^{n} \omega_{j} \frac{e^{-2 \pi \omega_{j}}}{1-e^{-2 \pi \omega_{j}}},
\end{aligned}
$$

we get

$$
A^{(1)}=\delta\left(\omega_{1}+\cdots \omega_{n+1}\right) e^{-1 / g_{s}} 2 \pi i g_{s} \omega \sinh (\pi \omega) 2^{n} \prod_{j=1}^{n} \sinh \left(\pi \omega_{j}\right)\left[\frac{1}{4 \pi^{2}}-\sum_{i=1}^{n} \frac{\omega_{i}}{4 \pi} \operatorname{coth}\left(\pi \omega_{i}\right)\right] .
$$

Eq. (2.9) would agree with the subleading order term in (2.4) if,

$$
\begin{gathered}
\sum_{\substack{i, j=1 \\
i<j}}^{n+1} f\left(\omega_{i}, \omega_{j}\right)+\sum_{i=1}^{n+1} g\left(\omega_{i}\right)+C=-i \sum_{j=1}^{n} \omega_{j}\left(1-\sum_{i=1}^{n} \pi \omega_{i} \operatorname{coth}\left(\pi \omega_{i}\right)\right) \\
\text { for } \omega_{n+1}=-\sum_{i=1}^{n} \omega_{i}, \quad \omega_{i}>0 \text { for } 1 \leq i \leq n,
\end{gathered}
$$

or, equivalently,

$$
\begin{aligned}
& \sum_{\substack{i, j=1 \\
i<j}}^{n} f\left(\omega_{i}, \omega_{j}\right)+\sum_{i=1}^{n} f\left(\omega_{i},-\omega_{1}-\cdots-\omega_{n}\right)+\sum_{i=1}^{n} g\left(\omega_{i}\right)+g\left(-\omega_{1}-\cdots-\omega_{n}\right)+C \\
= & -i \sum_{j=1}^{n} \omega_{j}\left(1-\sum_{i=1}^{n} \pi \omega_{i} \operatorname{coth}\left(\pi \omega_{i}\right)\right), \quad \text { for } \omega_{i}>0, \quad 1 \leq i \leq n .
\end{aligned}
$$




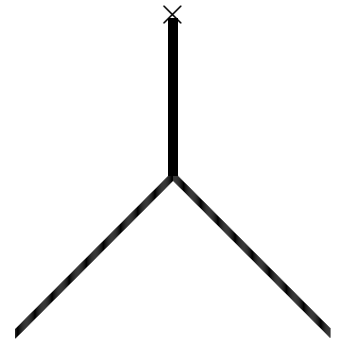

(a)

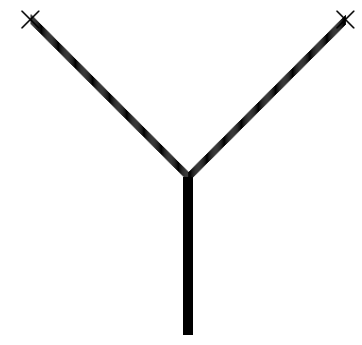

(b)

Figure 1. The Feynman diagrams contributing to the imaginary parts of $f\left(\omega_{1}, \omega_{2}\right)$ (figure (a)) and $g(\omega)$ (figure (b)). The thick lines denote closed string propagators and the $\times$ 's denote the closed string one point function on the disk.

\section{Divergences to imaginary parts}

The expressions for the functions $f\left(\omega_{1}, \omega_{2}\right)$ and $g(\omega)$, given in (1.2) and (1.3), are formally real, while the matrix model result (2.9) is purely imaginary. Therefore (2.11) looks wrong. However, the reality of $f$ and $g$ is misleading. As in any quantum field theory, a string amplitude is expected to acquire an imaginary part when the energy of a subset of external states exceeds the threshold of production of physical intermediate states. However, in the world-sheet description, whenever an amplitude is expected to acquire an imaginary part, the corresponding integral over the moduli space of Riemann surfaces diverges [17-23]. In the present case, the imaginary parts of $f$ and $g$, and the associated divergences in the world-sheet description, arise from the closed string degeneration associated with the Feynman diagrams shown in figure 1. In order to avoid these divergences, ref. [1] worked with imaginary energies where these divergences are absent, and verified (1.7) for $n=1$ in this domain numerically.

It is however possible to work with real energies and extract finite answers either by deforming the contour of integration over the moduli of Riemann surfaces into complex plane [16], or by using string field theory description of the amplitudes as integrals over loop momenta, and then deforming the momentum integration contours into the complex plane [15]. We shall illustrate this below by showing how to get finite results for $f\left(\omega_{1}, \omega_{2}\right)$ and $g(\omega)$ using the string field theory based approach, and also analytically computing the imaginary parts of these functions for real energies.

Let us begin by analyzing the divergent part of $f\left(\omega_{1}, \omega_{2}\right)$ coming from the $y \rightarrow 1$ region of (1.2). This contribution was analyzed in eq. (2.14), (2.15) and (2.18) of [1]. After carefully evaluating the overall normalization factors, and accounting for the fact that we are labelling the incoming and the outgoing energies by $-\omega_{1}$ and $\omega_{2}$ respectively, the relevant divergent part of $f\left(\omega_{1}, \omega_{2}\right)$ takes the form:

$$
\begin{gathered}
\frac{1}{\sinh \left(\pi\left|\omega_{1}\right|\right) \sinh \left(\pi\left|\omega_{2}\right|\right)} \int_{0}^{\infty} d P \int^{1} d y(1-y)^{-1+2 P^{2}-\left(\omega_{1}+\omega_{2}\right)^{2} / 2} 2^{-2 P^{2}+\left(\omega_{1}+\omega_{2}\right)^{2} / 2} \\
\mathcal{C}\left(\left|\omega_{1}\right| / 2,\left|\omega_{2}\right| / 2, P\right) \sinh (2 \pi P),
\end{gathered}
$$

where $\mathcal{C}\left(P_{1}, P_{2}, P_{3}\right)$ is the three point functions of primaries, carrying momenta $P_{1}, P_{2}$ and 
$P_{3}$, in $c=25$ Liouville theory [25, 26], normalized as in [27]. The $y$ integral diverges ${ }^{5}$ for $P^{2}<\left(\omega_{1}+\omega_{2}\right)^{2} / 4$. However string field theory tells us to replace the $y$-integral as follows $[10]$ :

$$
\int^{1} d y(1-y)^{-1+2 P^{2}-\left(\omega_{1}+\omega_{2}\right)^{2} / 2} \Rightarrow \int^{1-a} d y(1-y)^{-1+2 P^{2}-\left(\omega_{1}+\omega_{2}\right)^{2} / 2}+\frac{a^{2 P^{2}-\left(\omega_{1}+\omega_{2}\right)^{2} / 2}}{2 P^{2}-\left(\omega_{1}+\omega_{2}\right)^{2} / 2-i \epsilon},
$$

for any small but finite positive number $a$. The $i \epsilon$ has been introduced according to the $i \epsilon$ prescription for the propagators of string field theory, and at the end we have to take the limit $\epsilon \rightarrow 0^{+}$. In this limit, (3.2) is an identity for $2 P^{2}-\left(\omega_{1}+\omega_{2}\right)^{2} / 2>0$ but follows from the Feynman rules of string field theory for $2 P^{2}-\left(\omega_{1}+\omega_{2}\right)^{2} / 2 \leq 0$. It is also easy to check that the right hand side is independent of $a$ in the $\epsilon \rightarrow 0^{+}$limit. Therefore, making the replacement (3.2) in (3.1), one can extract finite results for the 'divergent' integral (3.1). One can even evaluate it numerically if desired keeping the energies $\omega_{1}$ and $\omega_{2}$ real.

Let us now discuss the computation of the imaginary part of $f\left(\omega_{1}, \omega_{2}\right)$. Since the integrand in the expression for $f\left(\omega_{1}, \omega_{2}\right)$ given in (1.2) is formally real, the only source of the imaginary part is the $i \epsilon$ in (3.2). Using the result,

$$
\frac{1}{2 P^{2}-\left(\omega_{1}+\omega_{2}\right)^{2} / 2-i \epsilon}=\frac{i \pi}{2\left|\omega_{1}+\omega_{2}\right|} \delta\left(P-\frac{\left|\omega_{1}+\omega_{2}\right|}{2}\right)+\text { real for } P>0
$$

in (3.2), and substituting this into (3.1), we get the following expression for the imaginary part of $f\left(\omega_{1}, \omega_{2}\right)$ :

$$
\begin{aligned}
f_{\text {imaginary }}\left(\omega_{1}, \omega_{2}\right)= & \frac{i \pi}{2 \sinh \left(\pi\left|\omega_{1}\right|\right) \sinh \left(\pi\left|\omega_{2}\right|\right)} \\
& \frac{1}{\left|\omega_{1}+\omega_{2}\right|} \sinh \left(\pi\left|\omega_{1}+\omega_{2}\right|\right) \mathcal{C}\left(\frac{\left|\omega_{1}\right|}{2}, \frac{\left|\omega_{2}\right|}{2}, \frac{\left|\omega_{1}+\omega_{2}\right|}{2}\right) .
\end{aligned}
$$

Using the result (see e.g. [27]),

$$
\mathcal{C}(a, b, a+b)=\mathcal{C}(a, a+b, b)=\mathcal{C}(a+b, a, b)=8 a b(a+b),
$$

we can express (3.4) as

$$
f_{\text {imaginary }}\left(\omega_{1}, \omega_{2}\right)=\frac{1}{2} i \pi \omega_{1} \omega_{2}\left\{\operatorname{coth}\left(\pi \omega_{1}\right)+\operatorname{coth}\left(\pi \omega_{2}\right)\right\} \operatorname{sign}\left(\omega_{1}+\omega_{2}\right) .
$$

Next we turn to the computation of $g(\omega)$. Our focus will be on extracting finite result from the $t \simeq 0$ region of (1.3) where the integral is apparently divergent. Comparing (1.3) of this paper with eqs. (2.27) of [1] and using the results of appendix A of [1], the divergent part of $g(\omega)$ from this region can be written in the form:

$$
\begin{gathered}
\frac{1}{\sinh (\pi|\omega|)} 2^{7 / 2} \pi \int_{0}^{\infty} d P_{1} \int_{0}^{\infty} d P_{2} \mathcal{C}\left(|\omega| / 2, P_{1}, P_{2}\right) \sinh \left(2 \pi P_{1}\right) \sinh \left(2 \pi P_{2}\right) \\
\int_{0}^{\infty} d s \int_{0}^{\frac{1}{4}} d x s^{1 / 2} \exp \left[-2 \pi s\left\{(1-2 x) P_{1}^{2}+2 x P_{2}^{2}-x\left(\frac{1}{2}-x\right) \omega^{2}\right\}\right],
\end{gathered}
$$

\footnotetext{
${ }^{5}$ In [1] this part of the analysis was carried out for $\omega_{1}+\omega_{2}=0$, and therefore they did not encounter this divergence.
} 
where the integration variables $s$ is related to $t$ in (1.3) by $s=1 / t$. Therefore the divergence near $t=0$ now arises from the region of large $s$. We have chosen the lower limit of $s$ integral to be 0 for definiteness, but this has no particular significance - we can change this to any other value at the cost of adding a finite contribution. Let us now change the integration variables from $(s, x)$ to

$$
t_{1}=2 \pi s(1-2 x), \quad t_{2}=4 \pi s x .
$$

We note further that for $x \leq 1 / 4$, we have $t_{2} \leq t_{1}$. We shall relax this by allowing $x$ integration to run over the range $0 \leq x \leq 1 / 2$ at the cost of multiplying the integrand by a factor of $1 / 2$. This reduces (3.7) to

$$
\begin{gathered}
\frac{1}{\sinh (\pi|\omega|)} \pi^{-1 / 2} \int_{0}^{\infty} d P_{1} \int_{0}^{\infty} d P_{2} \mathcal{C}\left(|\omega| / 2, P_{1}, P_{2}\right) \sinh \left(2 \pi P_{1}\right) \sinh \left(2 \pi P_{2}\right) \\
\int_{0}^{\infty} d t_{1} \int_{0}^{\infty} d t_{2}\left(t_{1}+t_{2}\right)^{-1 / 2} \exp \left[-t_{1} P_{1}^{2}-t_{2} P_{2}^{2}+\frac{t_{1} t_{2}}{t_{1}+t_{2}} \frac{\omega^{2}}{4}\right] .
\end{gathered}
$$

This integral diverges from the large $t_{1}, t_{2}$ region for $\omega>2\left(P_{1}+P_{2}\right)$, but there is no divergence from the region of large $t_{1}$ at fixed $t_{2}$ or vice versa. However, as has been discussed in detail in [15], this divergence can be attributed to the wrong use of Schwinger parametrization in a string field theory Feynman diagram. Ref. [15] also discusses how to extract finite answer from this apparently divergent integral. Comparing eq. (2.11) of [15] for $D=1$ to the sum of eqs. (2.4) and (2.5) of [15], we get the replacement rule:

$$
\begin{gathered}
(4 \pi)^{-1 / 2} \int_{A}^{\infty} d t_{1} \int_{A}^{\infty} d t_{2}\left(t_{1}+t_{2}\right)^{-1 / 2} \exp \left[\frac{t_{1} t_{2}}{t_{1}+t_{2}} M^{2}-\left(t_{1} m_{1}^{2}+t_{2} m_{2}^{2}\right)\right] \\
\Rightarrow \exp \left[A\left(M-m_{2}\right)^{2}-A m_{1}^{2}\right]\left(2 m_{2}\right)^{-1}\left\{M+m_{1}-m_{2}\right\}^{-1}\left\{m_{1}+m_{2}-M-i \epsilon\right\}^{-1} \Theta\left(M-m_{2}\right) \\
\quad+\int_{-\infty}^{\infty} \frac{d u}{2 \pi} \exp \left[-A\left\{u^{2}+m_{1}^{2}\right\}-A\left\{(u+i M)^{2}+m_{2}^{2}\right\}\right]\left(u^{2}+m_{1}^{2}\right)^{-1}\left\{(u+i M)^{2}+m_{2}^{2}\right\}^{-1},
\end{gathered}
$$

where $\Theta$ is the Heaviside function. The right hand side is a finite integral. Therefore, substituting this into (3.9) after replacing $m_{1}, m_{2}, M$ by $P_{1}, P_{2}, \omega / 2$ we can get finite result for (3.9).

The physical interpretation of (3.10) is as follows. The left hand side represents the contribution where we exress the two internal propagators in figure 1 by their Schwinger parameter representation and carry out the integration over the energies carried by these propagators. The right hand side represents the result of carrying out internal energy integrals directly, by deforming the energy integration contour to lie along the imaginary axis, and picking up residues from the poles that the contour crosses during this deformation. The external energy $\propto M$ is kept real. The first term on the right hand side is the residue at the pole that the contour crosses, while the second term represents the integration along the imaginary energy axis. The left hand side arises in the world-sheet theory, but string field theory tells us that the right hand side is the correct one when the two differ. For $M<m_{1}+m_{2}$, both sides are finite and (3.10) holds identically. 
Let us now compute the imaginary part of $g(\omega)$. Again, since the integrand for $g(\omega)$ in (1.3) is formally real, the only sources of imaginary parts of $g(\omega)$ are the $i$ 's on the right hand side of (3.10). The imaginary part of (3.10) is given by:

$$
i \pi\left(4 m_{1} m_{2}\right)^{-1} \delta\left(m_{1}+m_{2}-M\right)
$$

where we have used the fact that the term in the last line of (3.10) is real - this can be checked by making a $u \rightarrow-u$ transformation. Using (3.11) in (3.9), we get the imaginary part of $g(\omega)$ :

$$
\begin{aligned}
g_{\text {imaginary }}(\omega)= & \frac{i \pi}{2 \sinh (\pi|\omega|)} \int_{0}^{\infty} d P_{1} \int_{0}^{\infty} d P_{2} \mathcal{C}\left(|\omega| / 2, P_{1}, P_{2}\right) P_{1}^{-1} P_{2}^{-1} \\
& \sinh \left(2 \pi P_{1}\right) \sinh \left(2 \pi P_{2}\right) \delta\left(P_{1}+P_{2}-\frac{|\omega|}{2}\right) .
\end{aligned}
$$

Using (3.5) we can express this as

$$
g_{\text {imaginary }}(\omega)=\frac{i \pi}{2}|\omega|\left\{\omega \operatorname{coth}(\pi \omega)-\frac{1}{\pi}\right\}
$$

Using (3.6) and (3.13), it is now straightforward to verify that the imaginary part of the left hand side of (2.11) agrees with the matrix model answer given on the right hand side. Therefore (2.11) can now be written as an equation involving the real parts of $f$ and $g$ :

$$
\sum_{\substack{i, j=1 \\ i<j}}^{n} f_{\text {real }}\left(\omega_{i}, \omega_{j}\right)+\sum_{i=1}^{n} f_{\text {real }}\left(\omega_{i},-\omega_{1}-\cdots-\omega_{n}\right)+\sum_{i=1}^{n} g_{\text {real }}\left(\omega_{i}\right)+g_{\text {real }}\left(-\omega_{1}-\cdots-\omega_{n}\right)+C=0,
$$

for $\omega_{i}>0$. A class of solutions to (3.14) is provided by

$$
f_{\text {real }}\left(\omega_{i}, \omega_{j}\right)=\omega_{i} h\left(\omega_{j}\right)+\omega_{j} h\left(\omega_{i}\right), \quad g_{\text {real }}(\omega)=\omega h(\omega), \quad C=0
$$

for any function $h(\omega)$. It will be interesting to explore whether the results of string theory computation yields this form.

\section{Acknowledgments}

I wish to thank Bruno Balthazar, Victor Rodriguez and Xi Yin for useful discussions. This work was supported in part by the J. C. Bose fellowship of the Department of Science and Technology, India and the Infosys chair professorship.

Open Access. This article is distributed under the terms of the Creative Commons Attribution License (CC-BY 4.0), which permits any use, distribution and reproduction in any medium, provided the original author(s) and source are credited. 


\section{References}

[1] B. Balthazar, V.A. Rodriguez and X. Yin, ZZ Instantons and the Non-Perturbative Dual of $c=1$ String Theory, arXiv:1907.07688 [INSPIRE].

[2] S.R. Das and A. Jevicki, String Field Theory and Physical Interpretation of D 1 Strings, Mod. Phys. Lett. A 5 (1990) 1639 [InSPIRE].

[3] A.M. Sengupta and S.R. Wadia, Excitations and interactions in $d=1$ string theory, Int. J. Mod. Phys. A 6 (1991) 1961 [INSPIRE].

[4] D.J. Gross and I.R. Klebanov, Fermionic string field theory of $c=1$ two-dimensional quantum gravity, Nucl. Phys. B 352 (1991) 671 [INSPIRE].

[5] I.R. Klebanov, String theory in two-dimensions, in proceedings of String theory and quantum gravity '91, Trieste Spring School 1991, pp. 30-101 and Princeton University - PUPT-1271 (91/07,rec.Oct.) [hep-th/9108019] [INSPIRE].

[6] B. Balthazar, V.A. Rodriguez and X. Yin, Multi-Instanton Calculus in $c=1$ String Theory, arXiv: 1912.07170 [INSPIRE].

[7] B. Zwiebach, Closed string field theory: Quantum action and the B-V master equation, Nucl. Phys. B 390 (1993) 33 [hep-th/9206084] [INSPIRE].

[8] B. Zwiebach, Oriented open-closed string theory revisited, Annals Phys. 267 (1998) 193 [hep-th/9705241] [INSPIRE].

[9] C. de Lacroix, H. Erbin, S.P. Kashyap, A. Sen and M. Verma, Closed Superstring Field Theory and its Applications, Int. J. Mod. Phys. A 32 (2017) 1730021 [arXiv:1703.06410] [INSPIRE].

[10] A. Sen, String Field Theory as World-sheet UV Regulator, JHEP 10 (2019) 119 [arXiv: 1902.00263] [INSPIRE].

[11] P.V. Larocca and C. Maccaferri, BCFT and OSFT moduli: an exact perturbative comparison, Eur. Phys. J. C 77 (2017) 806 [arXiv: 1702.06489] [InSPIRE].

[12] A. Sen, Fixing an Ambiguity in Two Dimensional String Theory Using String Field Theory, JHEP 03 (2020) 005 [arXiv: 1908.02782] [INSPIRE].

[13] A. Sen, to appear.

[14] A. Sen, D-instanton Perturbation Theory, JHEP 08 (2020) 075 [arXiv:2002.04043] [INSPIRE].

[15] A. Sen, One Loop Mass Renormalization of Unstable Particles in Superstring Theory, JHEP 11 (2016) 050 [arXiv: 1607.06500] [INSPIRE].

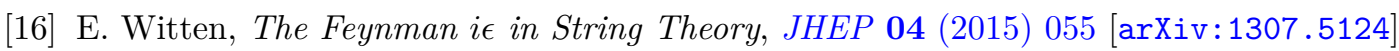
[INSPIRE].

[17] B. Sundborg, Selfenergies of Massive Strings, Nucl. Phys. B 319 (1989) 415 [InSPIRE].

[18] K. Amano and A. Tsuchiya, Mass Splittings and the Finiteness Problem of Mass Shifts in the Type-II Superstring at One Loop, Phys. Rev. D 39 (1989) 565 [inSPIRE].

[19] B. Sundborg, Infinite mass shifts of open superstrings as a possible signal of confinement, Nucl. Phys. B 338 (1990) 101 [InSPIRE].

[20] E. D'Hoker and D.H. Phong, Momentum analyticity and finiteness of the one loop superstring amplitude, Phys. Rev. Lett. 70 (1993) 3692 [hep-th/9302003] [INSPIRE]. 
[21] E. D'Hoker and D.H. Phong, Dispersion relations in string theory, Theor. Math. Phys. 98 (1994) 306 [Teor. Mat. Fiz. 98 (1994) 442] [hep-th/9404128] [INSPIRE].

[22] E. D'Hoker and D.H. Phong, The Box graph in superstring theory, Nucl. Phys. B 440 (1995) 24 [hep-th/9410152] [INSPIRE].

[23] A. Berera, Unitary string amplitudes, Nucl. Phys. B 411 (1994) 157 [inSPIRE].

[24] R. Pius and A. Sen, Cutkosky rules for superstring field theory, JHEP 10 (2016) 024 [Erratum ibid. 09 (2018) 122] [arXiv: 1604.01783] [INSPIRE].

[25] H. Dorn and H.J. Otto, Two and three point functions in Liouville theory, Nucl. Phys. B 429 (1994) 375 [hep-th/9403141] [INSPIRE].

[26] A.B. Zamolodchikov and A.B. Zamolodchikov, Structure constants and conformal bootstrap in Liouville field theory, Nucl. Phys. B 477 (1996) 577 [hep-th/9506136] [InSPIRE].

[27] B. Balthazar, V.A. Rodriguez and X. Yin, The $c=1$ string theory S-matrix revisited, JHEP 04 (2019) 145 [arXiv: 1705.07151] [INSPIRE]. 